\title{
Pic-Microcontroller Based Fully-Automatic Blood Pressure Monitor With Gsm Communication
}

\author{
R. A. Abdulkadir ${ }^{1}$, Umar Shuaibu $^{2}$, Aisha U. Musa ${ }^{3}$ \\ ${ }^{1,3}$ Department of Electrical Engineering, Kano University of Science and Technology, Wudil \\ ${ }^{2}$ Department of Electrical and Electronics Engineering, Sharda University, Greater Noida, India
}

\begin{abstract}
This paper described the development of PIC-Microcontroller based fully-automatic blood pressure monitoring system with GSM communication. Traditionally mercury Sphygmomanometers are used for blood pressure measurement; however the mercury sphygmomanometer method is manual and is mostly understood by expert. Later, Oscillometric devices often referred to as automatic devices are introduced; automatic blood pressure devices do not require observer participation beyond placing the cuff on the arm and noting the digital blood pressure readout. The objective of this paper is to enhance the existing automatic blood pressure devices by adding GSM communication facility so that health personnel can monitor a patient's blood pressure (BP) and heart rate from a remote location without requiring being physically present. PIC16F877A microcontroller is used to interface the blood pressure module to the GSM modem, the microcontroller control the overall operation of the device, blood pressure module is used to read the blood pressure and heart rate and communicates the result at 9600 baud rate to the microcontroller, which then display the result on an LCD and uses GSM for communicating the result by SMS directly to the health personnel's mobile phone. The system is physically constructed and its performance is tested using PIC microcontroller Development Board. The system operates successfully.
\end{abstract}

Key Words: Automatic Blood Pressure Monitoring, Heart rate, PIC-Microcontroller, GSM, LCD.

\section{INTRODUCTION}

The accurate measurement and control of blood pressure are key elements in the prevention of cardiovascular disease and stroke. As for adults (18 years and older) Blood pressure (BP) between 90-119 mm $\mathrm{Hg}$ systolic and 60-79 mm Hg diastolic are desired, BP between 120-139 mm Hg systolic and 80-89 $\mathrm{mm} \mathrm{Hg}$ diastolic is considered to be pre-hypertension condition, while elevated BP above $140 \mathrm{~mm} \mathrm{Hg}$ systolic and 90 $\mathrm{mm} \mathrm{Hg}$ diastolic is defined as hypertension [1]. High blood pressure usually does not have any symptoms; therefore, we need to have our blood pressure checked by specialist regularly. Aged people or physically handicapped people and the people suffering from some serious diseases experience so much difficulty when they need to go out for even small tasks. It is therefore of paramount importance to employ a system for monitoring blood pressure remotely without compromising the ordinary daily activities. Traditionally mercury Sphygmomanometers; consisting a column of mercury connected by rubber tubing to a manually inflated cuff are used for blood pressure measurement [2]. However, mercury has been found to be a potent human neurotoxin and also this manual method is mostly understood by specialist only. Later, aneroid and oscillometric devices were used as alternatives to mercury Sphygmomanometers. Aneroid Sphygmomanometers use mechanical parts to transmit the pressure in the cuff to a dial. As the cuff pressure rises, a thin brass corrugated bellows expands, triggering the movement of a pin resting on the bellows. This movement is amplified by a series of gears and transmitted to the dial where the blood pressure is read [3]. Oscillometric devices, often referred to as automatic devices, do not require observer participation beyond placing the cuff on the arm and noting the digital blood pressure readout[3].Present paper described the development of PIC-Microcontroller based fully-automatic blood pressure monitoring system with additional feature of GSM communication facility so that the doctor will be informed, and hence allowing for remote blood pressure monitoring.

\section{HARDWARE DESIGN}

The device comprises of blood pressure module to take the blood pressure and pulse rate measurements with local display attached to it, microcontroller (pic16f877a), 2x16 LCD and GSM modem for sending the result via an SMS. GSM mobile is used to receive the sent SMS.The components are interconnected as the block diagram shown in figure1. 


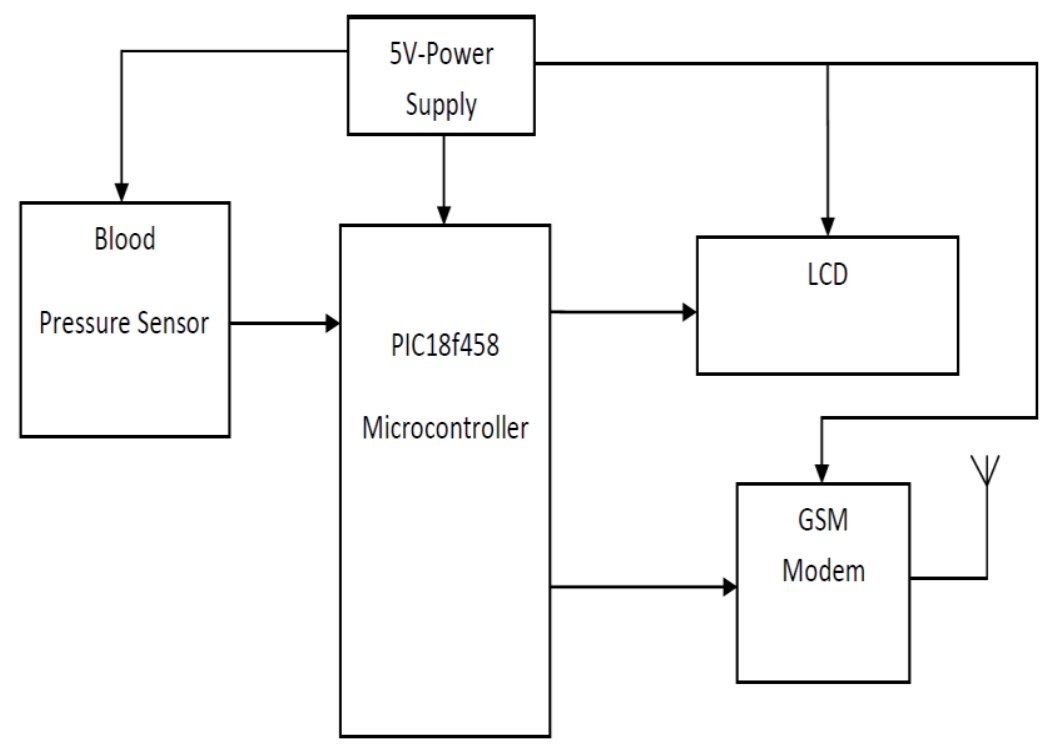

\subsection{PIC16F877A Microcontroller}

Figure 1: System Block Diagram

A microcontroller is a microcomputer on a single integrated circuit containing a processor, memory, and programmable input/output peripherals. PIC 16F877A is one of the most advanced microcontrollers. This controller is widely used for experimental and modern applications because of its low price, high quality, and ease of availability. The PIC 16F877A features almost all the components which modern microcontrollers normally have.

\subsection{Microcontroller-BP Module Interfacing}

The blood pressure module with serial output reads blood pressure and heart rate and communicates at 9600 baud rate( 8 bit data, No parity, and 1 stop bit).Blood Pressure \& Pulse readings are shown on the local display with serial out for external processing and display. The local display shows Systolic, Diastolic and Pulse Readings. It's so design to fit over wrist like a watch.

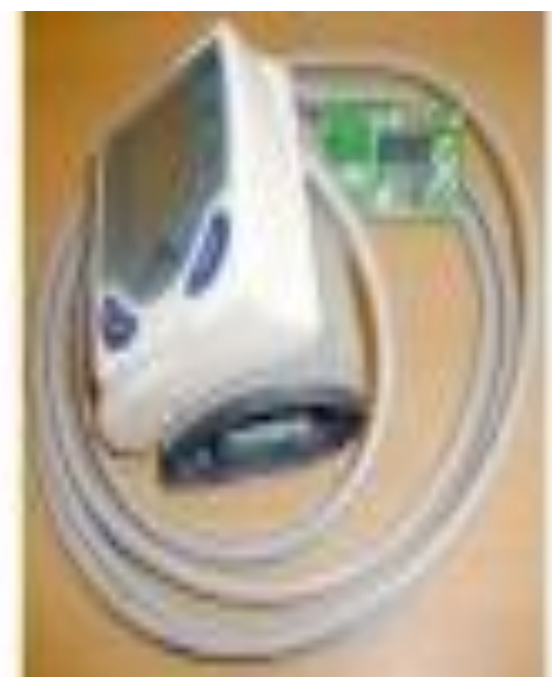

Figure 2: Blood Pressure Module with a serial communication port[4]

The BP module has been interfaced with the microcontroller through UART receiver pin (RC7/RX/DT pin of pic16f877A). The microcontroller is configured to communicate serially at 9600 baud rate. Microcontroller-BP Module interface is shown in figure. 


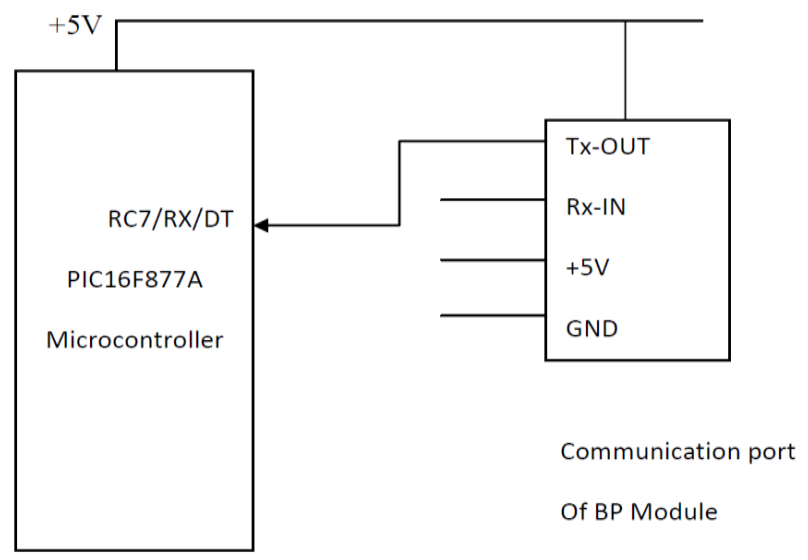

Figure 3: Microcontroller-BP Module Interface

\subsection{Microcontroller-LCD Interface}

A 2x16 LCD is used to display the result. LCD (Liquid Crystal Display) screen is an electronic display module. These modules are preferred over seven segments and other multi segment LEDs. The reasons being: LCDs are economical; easily programmable; have no limitation of displaying special \& even custom characters (unlike in seven segments), animations and so on. A 16×2 LCD can display 16 characters per line and there are 2 such lines. In this LCD each character is displayed in 5x7 pixel matrix. Figure below shows how the LCD has been interfaced with the microcontroller.The 8 pins of port B of the PIC microcontroller are used for interfacing the data pins of the LCD, the R/W pin is connected to ground ( $0 \mathrm{~V}$ level), the register select (RS) and Enable (EN) pins are connected to pin 28 (RD5) and pin 30 (RD7) of the microcontroller respectively.In the PIC programming for initializing the LCD the R/W pin is set to be low for writing the data, enable (EN) pin and register select pin (RS) are set to be high for writing the data. For sending a command the RS is set to be low, $\mathrm{R} / \mathrm{W}$ pin low and enable pin high.

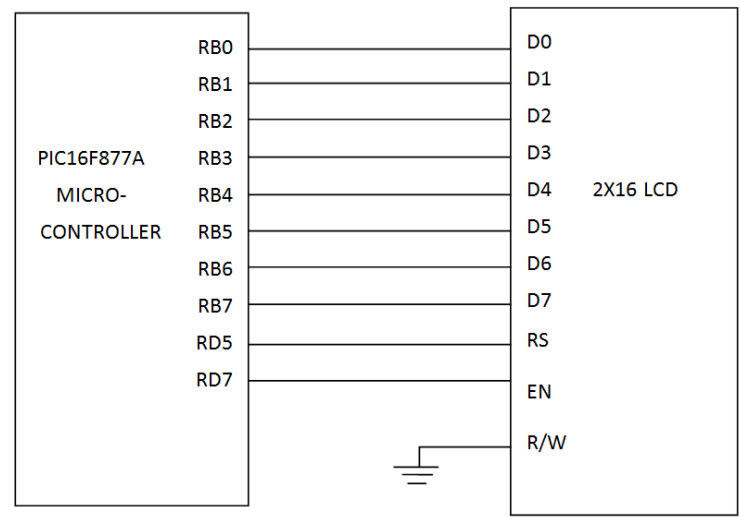

Figure 4: Microcontroller-LCD interface circuit

\subsection{Microcontroller-GSM Modem interface}

In the early 1980s, there was analogue communication technique, whereby each country developed its own system, this was problematic because the system work only within the boundary of each country.To this end, global system for mobile communication was developed by the conference of the European posts and telecommunication, prior to which exist a number of incompatible systems throughout Europe. The GSM was developed with the main aim of improving efficiency, international roaming, and compatibility with the international subscriber dialing and other telephone company[5].A GSM modem uses a SIM card, and operates over a subscription to a mobile operator, just like a mobile phone.GSM (Global system for mobile) uses a process called circuit switching to establish a path between two devices. Once the two devices are connected, a stream of digital data is conveyed between them. The GSM modem used in this device is SUNROM SIM 900D shown in figure5. 


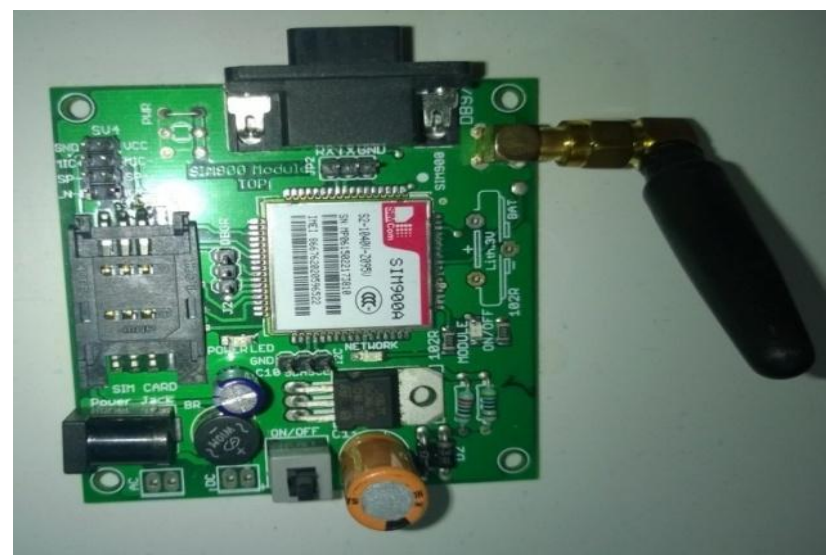

Figure 5: GSM MODEM (actual picture)

Table 1: Parameters and specification of GSM modem

\begin{tabular}{|c|c|c|}
\hline \multirow{5}{*}{$\begin{array}{c}\text { GSM } \\
\text { Modem }\end{array}$} & Frequency band & $\begin{array}{l}\text { Quad band } \\
\text { 850/900/1800/1900 }\end{array}$ \\
\hline & \begin{tabular}{|l|} 
Transmission power \\
\end{tabular} & $\begin{array}{l}\text { 2W @ @850/900 MHz } \\
1 \text { W @800/1900M }\end{array}$ \\
\hline & Band rate & 9600 \\
\hline & Power supply & $12 \mathrm{~V}, 1 \mathrm{~A}$ \\
\hline & \begin{tabular}{|l} 
Operating temperature \\
\end{tabular} & $-40^{\circ} \mathrm{C}$ to $85^{\circ} \mathrm{C}$ \\
\hline
\end{tabular}

The modem is directly interfaced with the PIC16f877A microcontroller. As per our requirement shown in Fig. 6 there is only 1 connection to use the modem. Rx pin of the modem is connected to the Tx pin of the microcontroller.

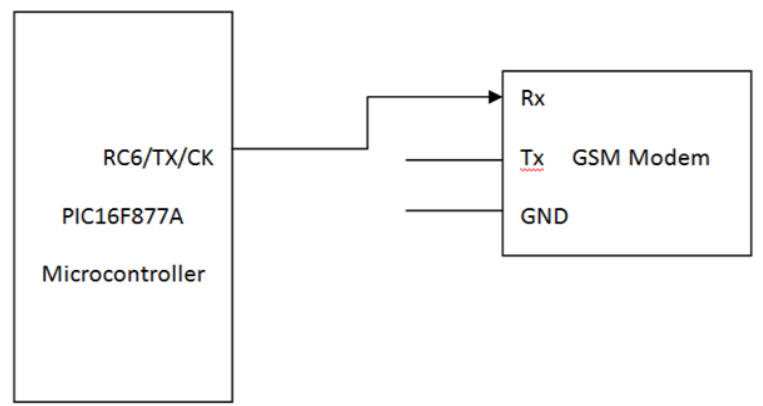

Figure 6: GSM Modem Interfacing with Microcontroller

\section{SOFTWARE DEVELOPMENT}

The software design involves writing and debugging the program and simulation. The software program was written in C programming language using mikroC PRO for PIC software.The system is simulated by using Proteus software.The system can successfully read the data from the BP module and display on the LCD using microcontroller.Since the Modem and the BP module are not available in Proteus, data transmitting and receiving functions are tested in Virtual terminals.

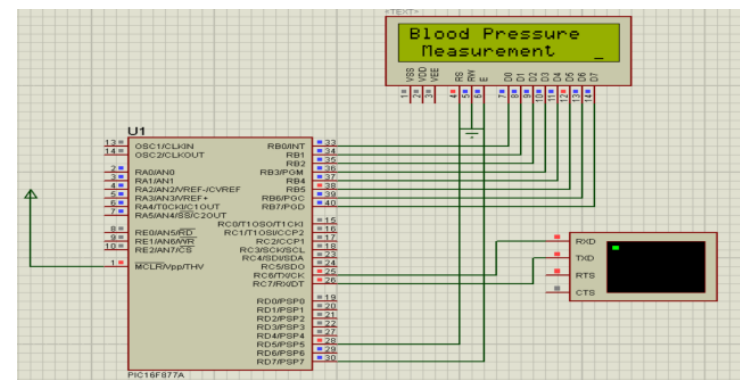

Figure 7: Proteus simulation 


\subsection{Operational Principle}

The system works on principle of fully-automated blood pressure monitoring system to measure and display systolic and diastolic pressures and pulse rate, then send the result to the healthcare or caregiver by SMS on GSM communication protocol. When the device is started the microprocessor initializes the memory, I/O pins, LCD and GSM module and feed SMS format into memory. "BLOOD PRESSURE MONITOR" is displayed on the LCD to inform the user that the device is ready. When the ON button of the Blood pressure module is pressed the cuff attached to the module automatically compressed and decompressed to take the measurements, and then display the result on local display attached to the module. The result is then communicated to the microcontroller via serial communication. The microcontroller display same result on the LCD (main display) and simultaneously transmits same to GSM modem which then send it by SMS to a predefined phone numbers (healthcare and caregiver contact numbers) for remote monitoring of the patience blood pressure (health condition).

\subsection{System Flowchart}

The flow chart of the software implementation of the system is illustrated in figure 8 .

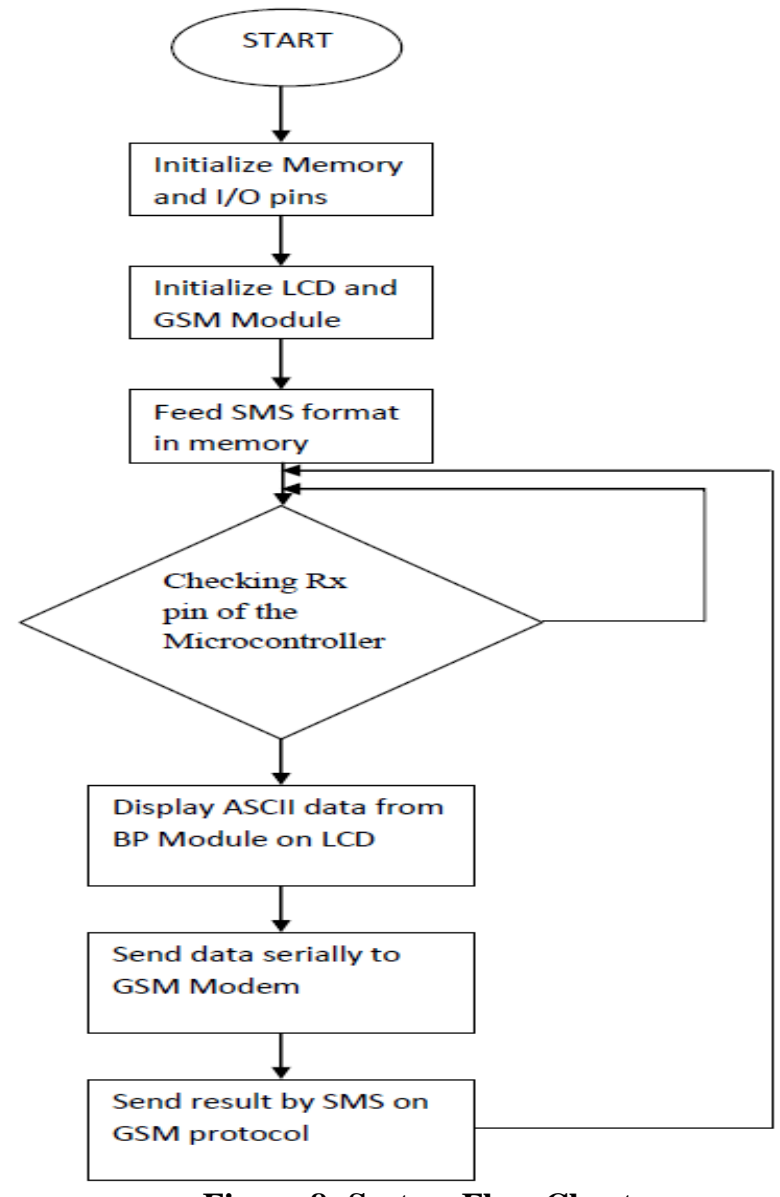

Figure 8: System Flow Chart

\section{TESTING AND EVALUATION}

The individual components were tested and found to be working properly. The GSM modem was tested with a PC, the GSM Modem was connected to a PC COM Port, a HyperTerminal (Windows tool for serial port communications) window was created with Baud rate 9600 and connected it to the COM Port to which GSM is connected, for any AT command typed in the HyperTerminal window the modem responded by sending "OK".

In this hardware construction, the BP module measures the systolic, diastolic pressures and pulse rate and display result on the local display attached to it. Then the measured data are transmitted to the microcontroller, the microcontroller will then display the result on the LCD.And then GSM modem transmits the data to the user's GSM mobile. As seen in the figures below, the constructed circuit can successfully display and transmit the data. Transmit and receive the condition (systolic, diastolic and pulse). 

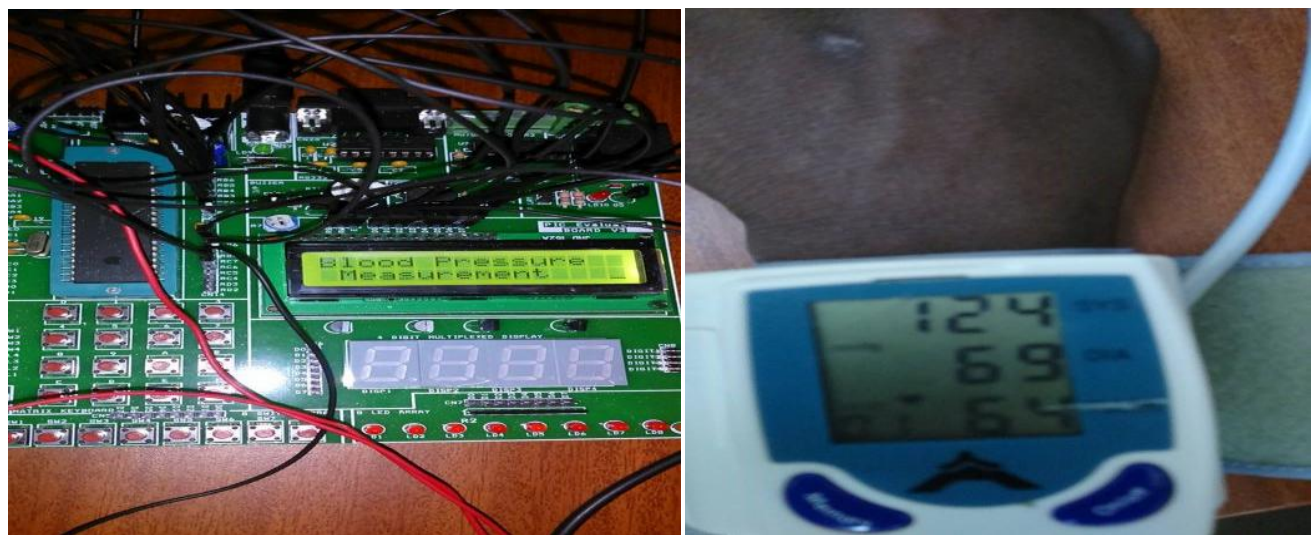

a) System Initializationb) Result on Local the Display
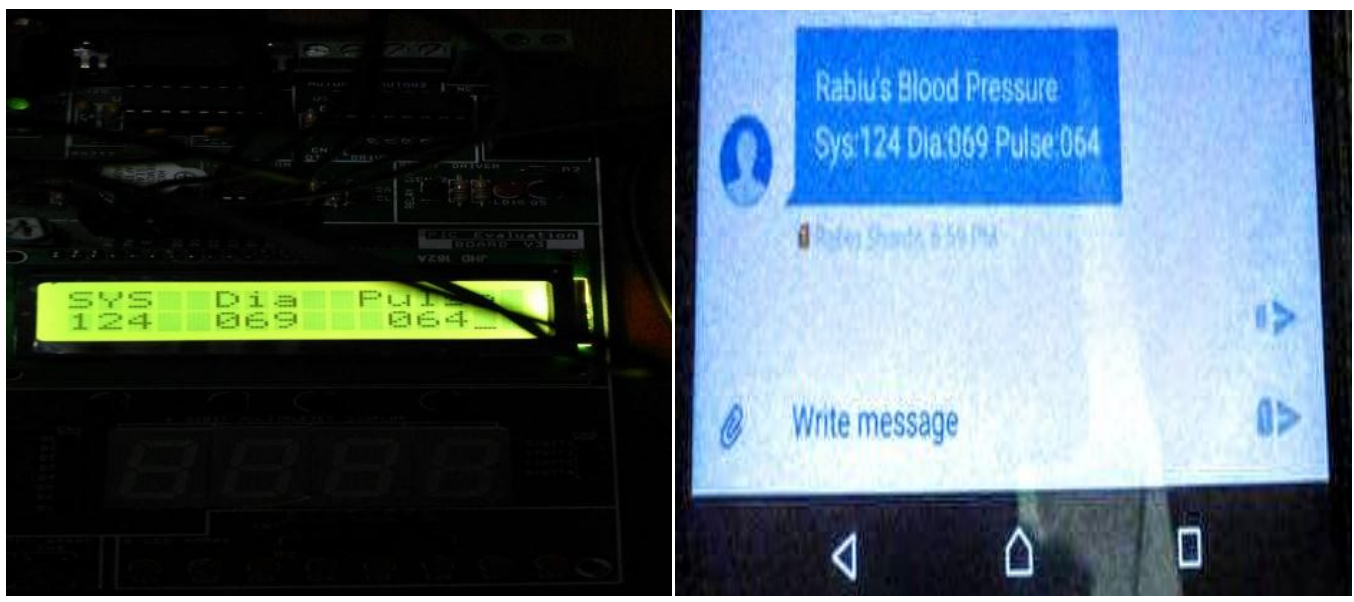

c) Result on the LCD d) Received SMS on the User's GSM mobile phone

Figure 9: Test Results of Hardware Unit

V.

\section{CONCLUSION}

Microcontroller based fully-automatic blood pressure monitor with GSM communication is implemented in this paper. The system allows healthpersonnel to monitor a patient's BP and heart rate from a remote location without requiring the physician to bephysically present to take the measurements. The system is physically constructed and its performance has been tested. The system operates successfully. Therefore, this proposed system can be practically used for remote monitoring of the patient blood pressure. The current implementation can be further improved. Future improvements to the system may include; a real time clock so that the date and time of recording can be sent along with the data, a keypad to change the recipient number when desired, and an alarm to alert the patient when an abnormality is found.

\section{REFERENCES}

[1] Feng, "Blood Pressure Meter Design Using Microchip's PIC24F Microcontroller and Analog Devices," Microchip Technology Inc, 2013.

[2] M.-C. Pan, P.-C. Li and Y.-R. Cheng, "Remote online machine condition monitoring system," Elsevier, vol. 41, no. 8, pp. 912-921, 2008.

[3] M. M. Susan Buchanan, "The Accuracy of Alternatives to Mercury Sphygmomanometers," H e a $1 \mathrm{t}$ h C a r e R e s e a r c h Co 11 a b o r a t i v e, Chicago, 2009.

[4] SUNROM Electronics, "Blood Pressure Sensor - Serial output," Febuary 2015. [Online]. Available: http://www.sunrom.com/p/blood-pressure-sensor-serial-output. [Accessed 2 June 2015].

[5] U. Shuaibu, H. K. Verma, R. A. Abdulkadir and C. Mohan, "DESIGN AND DEVELOPMENT OF INTELLIGENT ELECTRONICS TRAVELLING AID FOR VISUALLY IMPAIRED (IETA-V," International Journal of Research in Engineering and Technology, vol. 4, no. 11, pp. 341-348, 2015. 